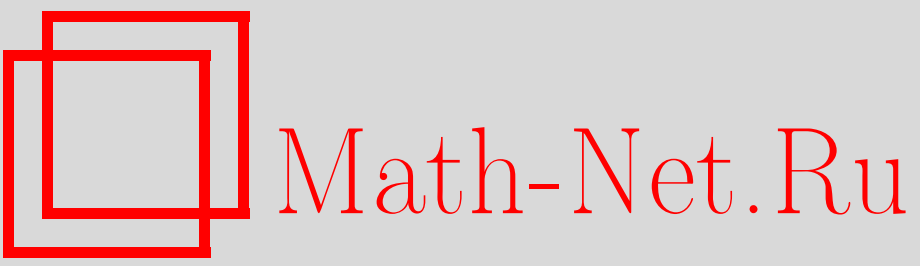

В. А. Зорич, В. М. Кесельман, Основная частота и конформный тип риманова многообразия, УМH, 2002, том 57, выпуск 2, 195-196

DOI: https://doi.org/10.4213/rm505

Использование Общероссийского математического портала Math-Net.Ru подразумевает, что вы прочитали и согласны с пользовательским соглашением

http://www.mathnet.ru/rus/agreement

Параметры загрузки:

IP: 54.147 .182 .235

26 апреля 2023 г., 15:02:23 


\title{
ОСНОВНАЯ ЧАСТОТА И КОНФОРМНЫЙ ТИП РИМАНОВА МНОГООБРАЗИЯ
}

\author{
В. А. ЗОРИЧ, В. М. КЕСЕЛЬМАН
}

I. Введение и формулировка основного результата. В этой заметке отмечена взаимосвязь конформного типа риманова многообразия и его основной частоты.

Рассматривается произвольное некомпак тное $n$-мерное риманово многообразие $\left(M^{n}, g\right)$ с краем (возможно, пустым). Здесь $g$ - исходная риманова метрика на $M^{n}$. Порождаемые этой метрикой $n$ - и $(n-1)$-меры Хаусдорфа (объем и площадь) будем обозначать через $\mu$ и $\sigma$ соответственно.

Емкость конденсатора. Подконденсатором на $M^{n}$ понимают упорядоченную пару $(F, G)$, где $G$ - открытое множество в $M^{n}$, а $F$ - компакт в $G$. Для произвольно заданного числа $p>1$ величина

$$
\operatorname{cap}_{p}(F, G):=\inf _{f} \int_{G}|\nabla f|^{p} d \mu
$$

называется $p$-емкостью конденсатора $(F, G)$. Здесь нижняя грань берется по всем гладким финитным в $G$ функциям $f$ таким, что $f \equiv 1$ на $F$ и $0 \leqslant f \leqslant 1$ в $G$.

Конформные метрики. Наряду с исходной метрикой, мы будем рассматривать на $M^{n}$ также римановы метрики $\widetilde{g}$ вида $\widetilde{g}=\gamma^{2} g$, где $\gamma$ - некоторая положительная регулярная функция на $M^{n}$. Такие метрики $\widetilde{g}$ назьваются конформно эквивалентнылми или, короче, конформными метрике $g$.

Легко видеть, что в случае $p=n$ при переходе от метрики $g$ к конформной ей метрике значение емкости произвольного конденсатора на $M^{n}$ не меняется. По этой причине величина сар ${ }_{n}(F, G)$ называется также конформной емкостью конденсатора $(F, G)$.

Конформный тип риманова многообразия. Для данного многообразия $M^{n}$ величина $\operatorname{cap}_{p}\left(F, M^{n}\right)$ либо положительна, либо равна нулю независимо от выбора невьрожденного континуума (отличного от точки связного компакта) $F$.

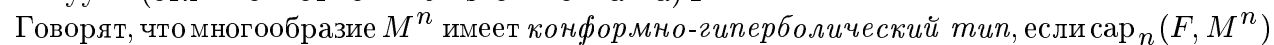
$>0$ для любого (или хотя бы одного) невырожденного континуума $F \subset M^{n}$; в противном случае говорят, что многобразие $M^{n}$ имеет конформно-параболический тип.

Ввиду отмеченной инвариантности конформной емкости, конформный тип многообразия определен конформно инвариантно.

Основная частота. Пусть $G$ - открытое множество в $M^{n}$. При заданном числе $p>1$ величина

$$
\lambda_{p}(G):=\inf _{f} \frac{\int_{G}|\nabla f|^{p} d \mu}{\int_{G}|f|^{p} d \mu}
$$

называетя $p$-основной частотой множеств $a$. З десь нижняя грань берется по всем гладким финитным в $G$ функциям $f \not \equiv 0$.

При $p=2$ величина $\lambda_{2}(G)$ является первым собственным значением оператора Лапласа однородной задачи Дирихлев $G$. В общем случае это же относится к уравнению $-\operatorname{div}\left(|\nabla f|^{p-2} \nabla f\right)=$ $\lambda|f|^{p-2} f$.

Рассматривая новую метрику $\widetilde{g}$ на $M^{n}$, для $p$-основной частоты множества $G$ будем также использовать обозначение $\lambda_{p}(G, \widetilde{g})$.

Теорема. Многообразие $\left(M^{n}, g\right)$ имеет конформно-гиперболический тип тогда и только тогда, когда существует конформная метрике $g$ метрика $\widetilde{g}$, для которой $\lambda_{n}\left(M^{n}, \widetilde{g}\right)>0$.

Следовательно, многообразие $\left(M^{n}, g\right)$ имеет конформно-параболический тип лишь тогда, когда в любой метрике $\widetilde{g}$, конформно эквивалентной его исходной метрике, $\lambda_{n}\left(M^{n}, \widetilde{g}\right)=0$.

Работа выполнена при поддержке Российского фонда фундаментальных исследований (грант № 02-01-01086). 
ЗАмечАниЕ. Если многообразие $\left(M^{n}, g\right)$ имеет конформно-гиперболический тип, то на нем также существуют конформные $g$ метрики $\widetilde{g}$, для которых $\lambda_{n}\left(M^{n}, \widetilde{g}\right)=0$.

II. Вспомогательные неравенства. Укажем некоторые понятия и соотношения, которые будут использованы при доказательстве сформулированных утверждений.

Константа Мазьи. Следуя работе [1], введем величину

$$
m_{p}(G):=\inf _{F \subset G} \frac{\operatorname{cap}_{p}(F, G)}{\mu(F)},
$$

связанную с $p$-основной частотой неравенствами:

$$
a_{p} m_{p}(G) \leqslant \lambda_{p}(G) \leqslant m_{p}(G),
$$

где $a_{p}=(p-1)^{p-1} p^{-p}$.

Левое (нетривиальное) неравенство в (1), по существу, доказано в [2]. Правое - непосредственно следует из определений $p$-емкости сар $p(F, G)$ и $p$-основной частоты $\lambda_{p}(G)$.

Изопериметрическое неравенство. Непрерьвная на $\mathbb{R}_{+}$функция $I(v)$ называется изопериметрической функцией многообразия $\left(M^{n}, g\right)$, если для любого предкомпактного открытого множества $G \subset M^{n}$ с гладкой границей $\partial G$ вьполняется неравенство:

$$
I(\mu(G)) \leqslant \sigma\left(\partial G \backslash \partial M^{n}\right),
$$

где $\partial M^{n}-$ край многообразия $M^{n}$

Если $I(v)$ - изопериметрическая функция многообразия $\left(M^{n}, g\right)$, то для константы Мазьи $m_{p}(G)$ произвольного открытого множества $G \subset M^{n}$ имеет место следующая оценка:

$$
\inf _{F \subset G} \frac{1}{\mu(F)}\left(\int_{\mu(F)}^{\mu(G)}(I(v))^{\frac{p}{1-p}} d v\right)^{1-p} \leqslant m_{p}(G) .
$$

Эта оценка непосредственно вытекает из соответствующего неравенства для емкости $\operatorname{cap}_{p}(F, G)$, полученного в [2].

III. Доказательства. Докажем утверждения, сформулированные в разделе I.

ДоКАЗАТЕЛЬСтво тЕОРЕмЫ. Предположим, что многообразие $\left(M^{n}, g\right)$ имеет конформногиперболический тип. Тогда, на основании резултата работы [3], на $M^{n}$ существует конформная $g$ метрика $\widetilde{g}$, для которой изопериметрическая функция $I(v)$ многообразия $\left(M^{n}, \widetilde{g}\right)$ имеет вид $I(v)=v$. Следовательно, в силу неравенства $(2)$, константа Мазьи $m_{n}\left(M^{n}\right)$ (относительно метрики $\widetilde{g})$ положительна. Отсюда, на основании левой части соотношения $(1), \lambda_{n}\left(M^{n}, \widetilde{g}\right)>0$.

С другой стороны, если многообразие $\left(M^{n}, g\right)$ имеет конформно-параболический тип, то для любой конформной $g$ метрики $\widetilde{g}$ константа Мазьи $m_{n}\left(M^{n}\right)=0$. Отсюда, в силу правой части соотношения (1), $\lambda_{n}\left(M^{n}, \widetilde{g}\right)=0$. Теорема доказана.

ДОКАЗАТЕЛЬСТВО ЗАмЕЧАНИЯ. Заметим преждевсего, что исходную метрику $g$ можно считать полной, так как этого всегда можно добиться конформным изменением метрики. Фиксируем теперь компактное исчерпание $\left\{F_{k}\right\}, k=1,2, \ldots$, многообразия $M^{n}$ и построим на $M^{n}$ гладкую положительную функцию $\gamma \geqslant 1$ такую, что $k \operatorname{cap}_{n}\left(F_{k}, M^{n}\right) \leqslant \int_{F_{k}} \gamma^{n} d \mu$ для всех $k$. Тогда в полной метрике $\widetilde{g}=\gamma^{2} g$ константа Мазьи $m_{n}\left(M^{n}\right)$ равна 0. Следовательно, для этой же метрики, ввиду (1), $\lambda_{n}\left(M^{n}, \widetilde{g}\right)=0$.

\section{СПИСОК ЛИТЕРАТУРЫ}

[1] A. Grigor'yan // Oper. Theory Adv. Appl. 1999. V. 109. P. 139-153. [2] В. Г. Мазья. Пространства С. Л. Соболева. Л.: Изд-во Ленинград. ун-та, 1985. [3] В. А. Зорич, В. М. Кесельман // Функц. анализ и его прил. 2001. Т. 35. № 2. С. 12-23.

Московский государственньй университет им. М. В. Ломоносова; Московский государственньй индустриальный университет
Принято редколлегией 23.01 .2002 